\title{
ZGDHu-1 and fludarabine have a synergistic effect on apoptosis of chronic lymphocytic leukemia cells
}

\author{
LIANNV QIU ${ }^{1}$, JINLIN LIU $^{1}$, ZHENNI WANG ${ }^{1}$, WEIXIAO HU ${ }^{2}$, QIANG HUANG ${ }^{3}$ and YONGLIE ZHOU ${ }^{1}$ \\ ${ }^{1}$ Department of Clinical Laboratory, Zhejiang Provincial People's Hospital; \\ ${ }^{2}$ College of Pharmaceutical Science, Zhejiang University of Technology; ${ }^{3}$ Department of Hematology, \\ Zhejiang Provincial People's Hospital, Hangzhou, Zhejiang 310004, P.R. China
}

Received April 8, 2015; Accepted May 22, 2015

DOI: $10.3892 /$ or.2015.4115

\begin{abstract}
Previously, it was demonstrated that the novel proteasome inhibitor N,N'-di-(m-methylphenyi)-3,6-dimethyl1,4-dihydro-1,2,4,5-tetrazine-1,4-dicarboamide (ZGDHu-1) possesses activity against chronic lymphocytic leukemia (CLL). In the present study, we attempted to assess whether this drug has a synergistic effect with fludarabine on the apoptosis of CLL cells. Annexin V/PI staining, mitochondrial membrane potential $(\Delta \Psi \mathrm{m})$ and reactive oxygen species (ROS) levels were examined by flow cytometry in short-term cell culture of blood cells from untreated newly diagnosed patients ex vivo. Expression of active caspase-3 and the Bcl-2/Bax ratio for determination of apoptosis were also investigated by flow cytometry and western blot analysis. Our results revealed that the ZGDHu-1 may induce the apoptosis of CLL cells through the mitochondrial pathway and its pro-apoptotic effect is CLL-specific, not affecting normal lymphocytes. Most importantly, a combination of ZGDHu-1 and a non-cytotoxic dose of fludarabine had a synergistic apoptotic effect. To some extent, caspase-3 activation may be involved in the mechanism of the ZGDHu-1 synergistic cytotoxic effect with fludarabine, as well as the cleavage of PARP, consequently leading to apoptosis. Notably, the rate of apoptosis caused by ZGDHu-1 alone or in combination with fludarabine was independent of prognostic markers of CLL disease such as ZAP-70 and CD38 expression or clinical Rai classification stage. In conclusion, ZGDHu-1 exhibited a significant synergistic effect with fludarabine to induce the apoptosis of CLL cells, which implies a possible clinical application.
\end{abstract}

\section{Introduction}

Chronic lymphocytic leukemia (CLL), the most common type of adult leukemia in Western countries, characterized

Correspondence to: Dr Liannv Qiu or Dr Yonglie Zhou, Department of Clinical Laboratory, Zhejiang Provincial People's Hospital, 158 Shangtang Road, Hangzhou, Zhejiang 310014, P.R. China

E-mail: qlv2012@126.com

E-mail: zhouyonglievip@163.com

Key words: chronic lymphocytic leukemia, ZGDHu-1, fludarabine, apoptosis, DNA damage by the accumulation of malignant $\mathrm{CD} 19^{+} \mathrm{CD} 5^{+}$cells, is a highly heterogeneous disease with variable prognoses and clinical course (1-3). Some patients never require treatment and are indolent, while other patients have aggressive disease and require intensive treatment soon after diagnosis $(4,5)$. Traditionally, symptomatic CLL patients can be effectively treated with fludarabine, glucocorticoids, alkylating agents or monoclonal antibodies. However, despite these therapeutic regimens, CLL disease is still incurable. Thus, the discovery of novel, less toxic and more effective drugs for CLL patients is in urgent need. Moreover, relapsed or refractory CLL patients have limited therapeutic treatment options.

To discover new drugs, the use of plant-derived substances or immunomodulatory drugs was reported to have a therapeutic effect in CLL treatment. Additionally, new candidates for CLL therapies include histone deacetylase inhibitors, Bcl-2 inhibitors and proteasome inhibitors have also been developed $(6,7)$. These latter agents may induce CLL cell apoptosis partly through pro-apoptotic and anti-apoptotic family members $(6,7)$. Among these drugs, fludarabine, an inhibitor of STAT1 activity and DNA synthesis inhibitor, is the most effective drug for the treatment of CLL disease, especially for routine treatment failure patients. However, toxic side effects in the clinic are extremely evident (8). The toxic effects of fludarabine include immunosuppression marked by a decrease in the $\mathrm{CD}^{+} / \mathrm{CD}^{+}$ratio, and the development of myelosuppression, opportunistic infections or gastrointestinal toxicities which include vomiting, nausea and hepatic lesions have also been reported (8).

ZGDHu-1 [N,N'-di-(m-methylphenyi)-3,6-dimethyl1,4-dihydro-1,2,4,5-tetrazine-1,4-dicarboamide] (Fig. 1) is a tetrazine compound (9), which has been reported by our group to exhibit antitumor activity (10). It has been identified as a potential proteasome inhibitor (11). It was demonstrated that ZGDHu-1 induces the apoptosis of B lymphocytes from CLL patients (12). In the present study, we investigated ZGDHu-1 used alone or combined with fludarabine in regards to the ex vivo apoptotic effects on CLL cells and normal lymphocytes derived from peripheral blood. We examined the apoptosis of CLL cells, loss of mitochondrial membrane potential $(\Delta \Psi \mathrm{m})$, phosphatidylserine (PS) translocation across the plasma membrane $(13,14)$ and accumulation of reactive oxygen species (ROS) (15). At the same time, the percentage 
of active caspase-3-expressing cells, intracellular Bcl-2 and Bax expression were also investigated. Subsequently, we also analyzed the correlation between these apoptotic effects with clinical indices, such as lactate dehydrogenase (LDH), ZAP-70 or CD38 expression, lymphocyte count, $\beta 2$-microglobulin level and Rai classification status.

\section{Materials and methods}

Patients. Twenty-five untreated, newly diagnosed CLL patients were enrolled. CLL diagnosis was carried out according to clinical examination, morphological and immunological criteria. After informed consent, peripheral blood cells were obtained from the CLL patients. The present study was approved by the Zhejiang Provincial People's Hospital Ethics Committee. Patient characteristics are summarized in Table I.

Reagents and instruments. ZGDHu-1 compound (Fig. 1) with a purity of $>95 \%$ was kindly provided by Dr Wei-Xiao $\mathrm{Hu}$ (Pharmaceutical College of Zhejiang University of Technology, China) as previously reported (12). ZGDHu-1 was dissolved in dimethylsulfoxide (DMSO) as a stock solution $(1 \mathrm{mg} / \mathrm{ml})$ and stored at $-20^{\circ} \mathrm{C}$. Antibodies against Bcl-2 (SC-7382), Bax (SC-2774), caspase-3 (SC-9746), $\beta$-actin (SC-4967) for western blot analysis were purchased from Cell Signaling Technology (Beverly, MA, USA). Fludarabine, DMSO, 3-(4,5-dimethylthiazol-2-yl)-2,5-diphenyltetrazolium bromide (MTT), dihydrorhodamine-123 (DHR), broad spectrum caspase inhibitor benzyloxycarbonyl-Val-Ala-Aspfluoromethylketone (Z-VAD-fmk) and Ficoll-Hypaque liquid were purchased from Sigma-Aldrich (St. Louis, MO, USA) as previously reported (12). The apoptosis kit of propidium iodide (PI), Annexin V and the IntraPrep ${ }^{\mathrm{TM}}$ permeabilization kit were all from Immunotech Company (Marseille, France). JC-1 (5,5',6,6'-tetrachloro-1,1',3,3'-tetrethyl benzimidalyl carbocyanine iodide) was purchased from BioTeam Inc. Company as previously reported (12). CLL cells were stained with the following mAbs: anti-CD19-PerCP CY 5.5 (ID3) and anti-CD5-APC (53.-7.3) (both from BD Pharmingen, San Diego, CA, USA), anti-ZAP-70 Alexa Fluor ${ }^{\circledR} 488$ (SBZAP) and anti-CD38-FITC (T16; both from Immunotech), anti-active caspase-3-PE (C92-605; BD Pharmingen), anti-Bax-FITC (SC20067; Santa Cruz Biotechnology, CA, USA); and anti-Bcl-2-PE (Bcl-2/100; BD Pharmingen). Cells were analyzed with Navios FACS (Beckman Coulter, Miami, FL, USA).

Cell viability assay. Cell viability was analyzed with an MTT assay as previously described (16). Cells (at a density of $\left.5 \times 10^{5} / \mathrm{ml}\right)$ were incubated with ZGDHu-1 (50, 100, 150, 200 and $250 \mathrm{ng} / \mathrm{ml}$ ) alone or in combination with fludarabine $(1 \mu \mathrm{g} / \mathrm{ml})$ on 96 -well plates for $72 \mathrm{~h}$. The control group was incubated only with drug-free medium with $0.05 \%$ DMSO solution (v/v). Then, MTT $(5 \mathrm{mg} / \mathrm{ml})$ was added to each well and incubated for $4 \mathrm{~h}$ at $37^{\circ} \mathrm{C}$, the medium was aspirated and then $150 \mu \mathrm{l}$ DMSO solution was added to each well. The plate was then measured by the M680 microplate reader (Bio-Rad, Hercules, CA, USA) at a reference $630 \mathrm{~nm}$ wavelength and a test $570 \mathrm{~nm}$ wavelength. All experiments were performed in triplicate and repeated at least three times. The cell viability was expressed as a percentage of the DMSO-treated control samples as previously reported (12).

Lymphocyte purification and culture. EDTA-K2 anticoagulant blood samples were obtained from the CLL patients and healthy controls during a routine diagnosis at the Zhejiang Provincial People's Hospital. B lymphocytes were isolated immediately by using Ficoll gradient centrifugation. After a 1-h incubation at $37^{\circ} \mathrm{C}$, in a $5 \% \mathrm{CO}_{2}$ condition, adhesive mononuclear cells were removed. The non-adherent lymphocytes were washed with Hank's solution (Biochrom, Berlin, Germany). Then the $\mathrm{T}$ lymphocytes were removed using anti-CD3 Dynabeads ${ }^{\circledR}$ (Dynal, Merseyside, UK). The B lymphocytes were further purified by flow cytometric sorting based on CD19 antibody staining (Immunotech, Coulter, USA) as previously reported (12). B lymphocytes were then counted in a Neubauer Counting Chamber with trypan blue to exclude dead cells. The cells were then resuspended in RPMI-1640 medium with $10 \%$ fetal bovine serum (FBS), $2 \mathrm{mM}$ glutamine, $100 \mathrm{U} / \mathrm{ml}$ penicillin $\mathrm{G}$ and $0.1 \mathrm{mg} / \mathrm{ml}$ streptomycin (Sigma-Aldrich) in $75 \mathrm{~cm}^{3}$ flasks at a density of $1-4 \times 10^{6} / \mathrm{ml}$ and cultured at $37^{\circ} \mathrm{C}$ in $5 \% \mathrm{CO}_{2}$ as previously reported (12). The cells were further divided into 4 groups. The first group served as the control without any treatment. The second group was treated with $100 \mathrm{ng} / \mathrm{ml} \mathrm{ZGDHu}-1$ for 0-5 days. The third group was treated with fludarabine $(1 \mu \mathrm{g} / \mathrm{ml})$ for $0-5$ days. The fourth group was treated with both ZGDHu-1 (100 ng/ml) and fludarabine $(1 \mu \mathrm{g} / \mathrm{ml})$ for 0-5 days. At the end of each time-point, the cells were harvested for FACS analysis or lysed for western blot analyses.

FACS. CLL cells were stained with the following mAbs: anti-CD19-PerCP CY 5.5 (ID3), anti-CD5-APC (53.-7.3), anti-ZAP-70 Alexa Fluor ${ }^{\circledR} 488$, anti-CD38-FITC (T16), anti-active caspase-3-PE(C92-605), anti-Bax-FITC (SC20067) and anti-Bcl-2-PE (Bcl-2/100). The stained cells were analyzed with NAVIOS FACS. Then 10,000 cells for each sample were counted and then CD19 and CD5 antibody staining was carried out and the $\mathrm{CD} 19^{+} \mathrm{CD}^{+}$double-positive cell population was gated for the following analysis. For the apoptosis detection, the percentage of Annexin V-positive (+) and PI-negative (-) cells was detected using the Annexin V kit. The mitochondrial potential $(\Delta \Psi \mathrm{m})$ was measured with JC-1 dye. The intracellular accumulation of ROS was assessed with the fluorescent dye DHR. The percentage of active caspase-3-positive cells in the control cells was calculated. The Bcl-2 and Bax expression was examined for each sample. Then the Bcl-2/Bax ratio for the $\mathrm{CD} 19^{+} \mathrm{CD}^{+}$cells was calculated. The $\mathrm{CD} 19^{+} \mathrm{CD}^{-}$populations were regarded as non-leukemic cells. To determine the frequency of prognostic factors, the percentage of CD38 and ZAP-70 was detected for each sample. Patients were defined as ZAP-70positive when the ZAP-70 expression was $>20 \%$ in leukemic cells. Patients were defined as CD38-positive, when the CD38 expression was at least $20 \%$ in leukemic cells.

Western blot analysis. The treated CLL and control cells were collected and lysed in buffer contained $20 \mathrm{mM}$ Tris- $\mathrm{HCl}$ (pH 7.5), 1 mM EDTA, $150 \mathrm{mM} \mathrm{NaCl}, 50 \mathrm{mM} \mathrm{NaF}, 10 \mathrm{mM}$ sodium pyrophosphate, $1 \mathrm{mM}$ sodium orthovanadate, $2 \mathrm{mM}$ 
Table I. Characteristics of the CLL patients.

\begin{tabular}{lc}
\hline Characteristics & Data \\
\hline Gender, $\mathrm{n}$ & \\
Female & 8 \\
Male & 17 \\
Age (years) & \\
Mean & 67.2 \\
Range & $58-89$ \\
Rai, n & \\
0 & 5 \\
I & 6 \\
II & 8 \\
III & 6 \\
Lymphocyte count $\left(x 10^{9} / 1\right)$ & \\
Mean & 21.6 \\
Range & $12.6-73.4$ \\
CD38 ${ }^{+}, \mathrm{n}(\%)$ & $6(24)$ \\
ZAP-70 ${ }^{+}, \mathrm{n}(\%)$ & $8(32)$
\end{tabular}

phenylmethylsulfonyl fluoride, $0.5 \%$ Triton-X and protease inhibitor cocktail (Pierce, Rockford, IL, USA) on ice for $30 \mathrm{~min}$. Then, the same amount of proteins was loaded and separated by SDS-PAGE gel, transferred to nitrocellulose membranes at $100 \mathrm{~V}$ for $2 \mathrm{~h}$ and then blocked with PBS solution with 5\% non-fat milk for $1 \mathrm{~h}$. After PBS washing, the membrane was incubated with the primary monoclonal antibodies against human Bcl-2, Bax and caspase-3 (1:1,000 dilution) for $1 \mathrm{~h}$, respectively. The $\beta$-actin expression was used as the control. Then, the rabbit anti-mouse IgG antibodies (1:1,000 dilution) were used as the secondary antibody. The ECL kit (Pierce) and the GDS-8000 imaging system (UVP, Upland, CA, USA) were used for the visualization of the immunoreactive bands.

Evaluation of the combination index. A combination index (CI) calculated based on the Chou-Talalay method was used to evaluate the synergism between ZGDHu-1 and fludarabine (2,17-19). The following formula was used: $\mathrm{CI}=$ (sum of single agent treatment/specific apoptosis of combined treatment). The percentage of specific apoptosis was examined by using the following formula: Specific apoptosis $=(\mathrm{drug}$ induced apoptosis - spontaneous apoptosis $) /(100$ - spontaneous apoptosis) $\mathrm{x} 100 \%$. $\mathrm{CI}<1, \mathrm{CI}=1$ and $\mathrm{C}>1$ were regarded as synergistic, additive or infra-additive, respectively $(20,21)$. The percentage of active caspase- 3 cells was estimated for these populations. In the present study, 7 of the 25 patients for whom the non-leukemic cells were higher than $10 \%$ in the peripheral blood were analyzed.

Statistical analysis. Data from individual experiments are presented as mean \pm SD. The statistical analysis was carried out using SPSS10.0 software. The Wilcoxon test was used for two dependent variables; Mann-Whitney U and Spearman's tests were used for two independent group and two variable correlations, respectively. $\mathrm{P}<0.05$ was considered to indicate a statistically significant result.

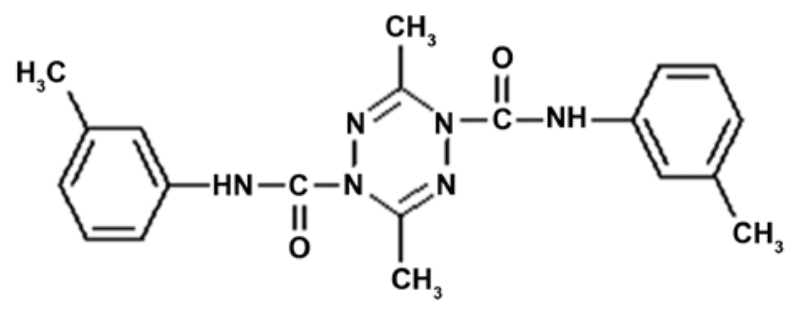

Figure 1. Molecular structure of ZGDHu-1.

\section{Results}

Effects of ZGDHu-1 and fludarabine on the viability of CLL cells. To investigate the synergistic effect of fludarabine and ZGDHu-1, we used the classical MTT assay to evaluate the cytotoxic effects of the two drugs. Firstly, we screened the minimal fludarabine cytotoxic concentration. After a series of preliminary studies with the aim to reduce the in vitro cytotoxicity above controls at a mean level of $<10 \%$ after a 3-day treatment, a $1 \mu \mathrm{g} / \mathrm{ml}$ concentration of fludarabine was regarded as being non-cytotoxic. Consistent with previous studies (22), fludarabine did not show significant cytotoxicity at the concentration of $1 \mu \mathrm{g} /$ $\mathrm{ml}$ compared to the controls $(5.24 \pm 1.33 \%)$. But when concentrations of 2 and $2.5 \mu \mathrm{g} / \mathrm{ml}(30.5 \pm 8.05 \% ; 41.7 \pm 7.25 \%$, respectively) were used, its cytotoxicity was significantly increased (Fig. 2). Furthermore, ZGDHu-1 treatment also caused an increase in the cytotoxicity to CLL cells in a dose-dependent manner; $5.8 \pm 1.34 \%$ at $50 \mathrm{ng} / \mathrm{ml}$ to $47.8 \pm 8.74 \%$ at $250 \mathrm{ng} / \mathrm{ml}$ on day 3 (Fig. 2). We then choose the optimal concentrations of fludarabine $(1 \mu \mathrm{g} / \mathrm{ml})$ and ZGDHu-1 (100 ng/ml). Notably, the minimal synergistic cytotoxic concentration was observed at ZGDHu-1 (100 ng/ml) and fludarabine $(1 \mu \mathrm{g} / \mathrm{ml})$, which was slightly increased when compare with ZGDHu-1 (100 ng/ml) and fludarabine $(1 \mu \mathrm{g} / \mathrm{ml})$ alone $(5.24 \pm 1.33 \%$ for fludarabine; $20.5 \pm 4.56 \%$ for ZGDHu-1; $39.5 \pm 7.45 \%$ for fludarabine + ZGDHu-1). Thus, in the following synergistic effect studies, the treatment duration was set at 3 days at a concentration of $100 \mathrm{ng} / \mathrm{ml}$ for ZGDHu-1 and $1 \mu \mathrm{g} / \mathrm{ml}$ for fludarabine.

Combination of ZGDHu-1 and fludarabine induces the apoptosis of CLL cells. To better support the MTT assay in regards to cell death triggered by ZGDHu-1 alone and in combination with fludarabine, CLL cells were evaluated for apoptosis by FACS analysis following Annexin-V and PI staining. As shown in Fig. 3, the apoptotic cell population was significantly increased after the CLL cells were treated with ZGDHu-1 and/or fludarabine on day 3 compared to the control (no treatment). Notably, the combination of ZGDHu-1 and fludarabine significantly increased the apoptotic population when compared to this population in the cells treated with ZGDHu-1 or fludarabine alone. To further investigate whether ZGDHu-1 and fludarabine induced CLL cell apoptosis through the caspase-dependent pathways, we treated CLL cells with ZGDHu-1 and/or fludarabine in the presence of the broad spectrum caspase inhibitor, Z-VAD-fmk. We found that the apoptotic cell population was significantly reduced after pretreatment with Z-VAD-fmk (Fig. 3A and B). These data indicate that ZGDHu-1 induced cell apoptosis through the caspase-dependent pathway. 


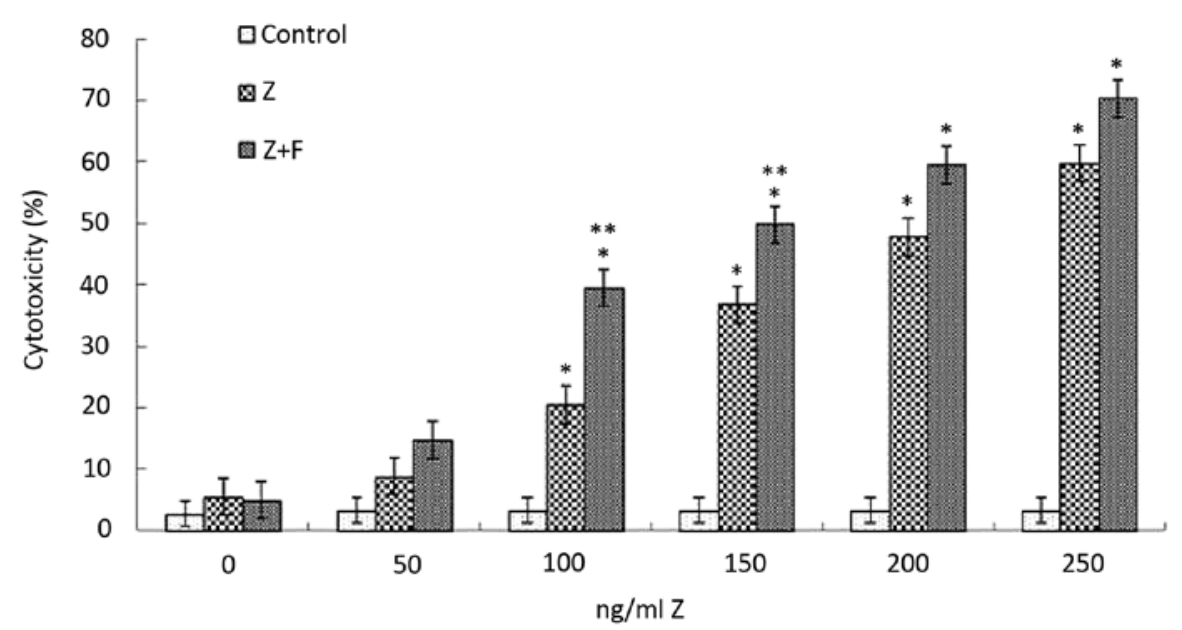

Figure 2. Synergistic cytotoxic effect of ZGDHu-1 and fludarabine in CLL cells. Purified CLL cells were cultured with ZGDHu-1 (from 50 to $250 \mathrm{ng} / \mathrm{ml}$ ) in the presence or absence of low-dose fludarabine $(1 \mu \mathrm{g} / \mathrm{ml})$ for $72 \mathrm{~h}$. Cytotoxicity was determined by the MTT assay. Low doses were defined as a level of cytotoxicity $<10 \%$ above the untreated controls. Experiments were carried out in triplicate and the results are expressed as mean $\pm \mathrm{SD}, \mathrm{n}=3$; ${ }^{*} \mathrm{P}<0.05$ vs. respective controls of triplicate wells. "* $\mathrm{P}<0.05$ represent ZGDHu-1 with fludarabine vs. ZGDHu-1 alone. F, $1 \mu \mathrm{g} / \mathrm{ml}$ fludarabine; Z, ZGDHu-1.

A

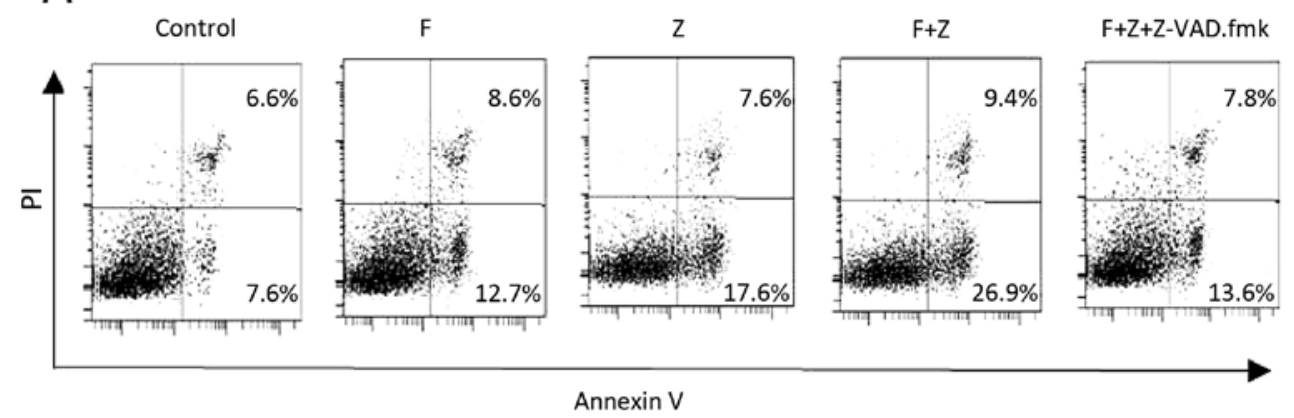

B

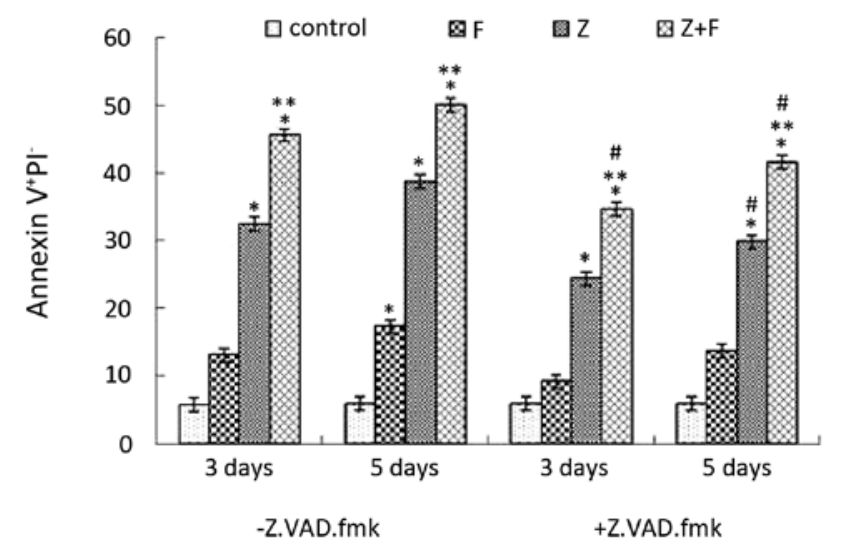

Figure 3. Apoptosis of CLL cells following treatment with ZGDHu-1 and fludarabine in vitro. (A) FACS histograms representing the mean of 10 individual experiments are shown. Control, 0.05\% DMSO drug-free medium (v/v); F, $1 \mu \mathrm{g} / \mathrm{ml}$ fludarabine; Z, $100 \mathrm{ng} / \mathrm{ml} \mathrm{ZGDHu}-1$. (B) Purified CLL cells were cultured in $0.05 \%(\mathrm{v} / \mathrm{v})$ DMSO drug-free medium (control) or with ZGDHu-1, fludarabine alone or a combination of ZGDHu-1 and fludarabine in the presence or absence of the broad spectrum caspase inhibitor Z-VAD-fmk for 3 or 5 days. The Annexin V assay was used for detection of early apoptotic cells. The results are expressed as means $\pm \mathrm{SD}, \mathrm{n}=10,{ }^{*} \mathrm{P}<0.01$ vs. control, respectively. ${ }^{* *} \mathrm{P}<0.05$ represent ZGDHu-1 with fludarabine vs. ZGDHu-1 alone. ${ }^{\#} \mathrm{P}<0.05$ represent $-\mathrm{Z} . \mathrm{VAD} . \mathrm{fmk}$ vs. +Z.VAD.fmk.

Effect of the combination of ZGDHu-1 and fludarabine on the mitochondrial pathway through the change in ROS. As we know, ROS play an important role in the mitochondrial pathway during cell apoptosis (15). The ROS level in CLL cells was examined following treatment with ZGDHu-1 alone or in combination with fludarabine. In the present study, the
ROS level was detected by DHR staining and FACS method. As shown in Fig. 4A and B, following ZGDHu-1 treatment alone and in combination with fludarabine on day 3 and 5 , the ROS levels were significantly increased (all $\mathrm{P}<0.05$ ). However, treatment with fludarabine for 3 or 5 days did not significantly increase the ROS level in the CLL cells. Additionally, the 
A

10

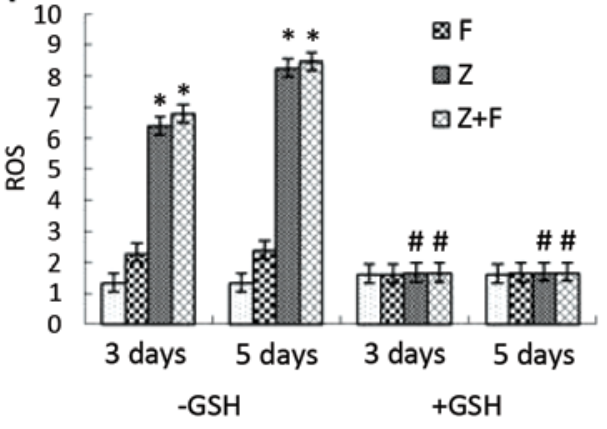

C

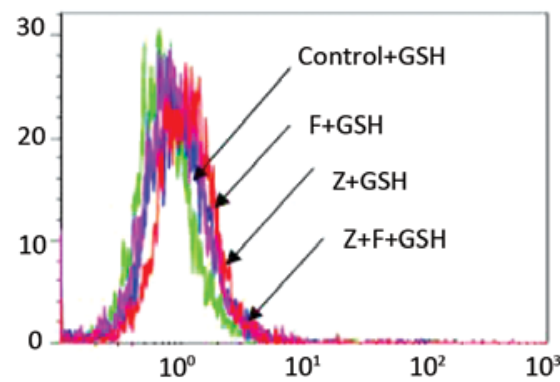

B

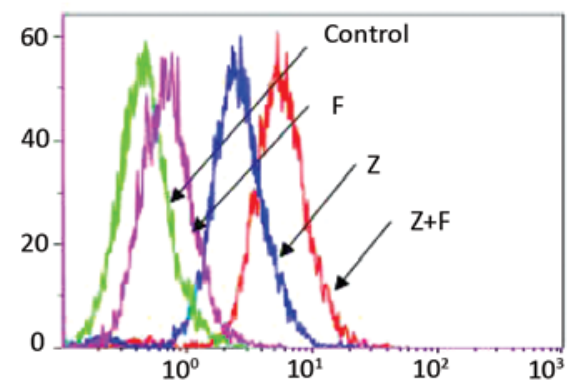

D

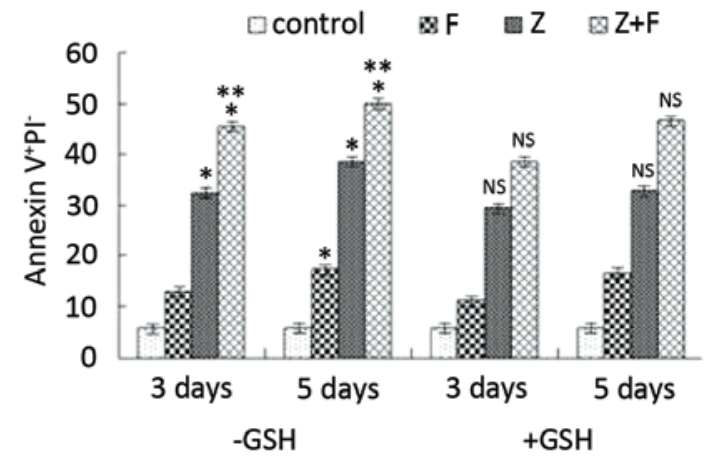

E

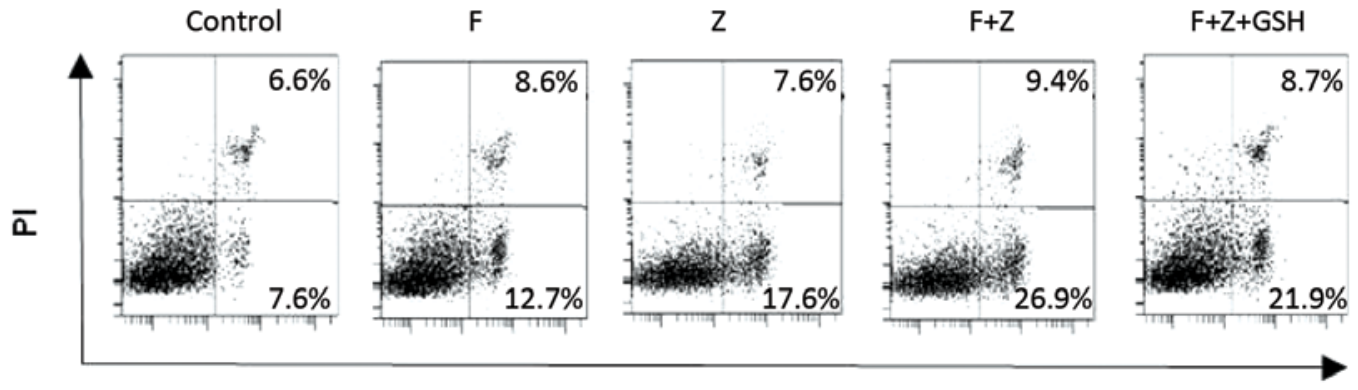

Annexin V

Figure 4. Effect of ZGDHu-1 and fludarabine on the level of ROS and apoptosis in CLL cells. (A) Purified CLL cells were cultured in 0.05\% (v/v) DMSO drugfree medium (control) with ZGDHu-1 alone, fludarabine alone or with the combination of ZGDHu-1 and fludarabine in the presence or absence of GSH for 3 and 5 days. DHR staining and flow cytometry were used for ROS measurement. Data are presented as the means \pm SD, n=10. "P<0.05 between ZGDHu-1 and ZGDHu-1 + GSH; "P $<0.05$ between treatment and control. (B) The mean fluorescence was enhanced by ZGDHu-1 alone or the combination of ZGDHu-1 and fludarabine in comparison to the control. Representative data of 10 independent experiments following exposure to ZGDHu-1, fludarabine alone or in combination with ZGDHu-1 for 3 days in the (B) absence or (C) in the presence of $100 \mu \mathrm{M} \mathrm{GSH}$. (D) Effect of GSH on the ZGDHu-1-induced apoptosis of CLL cells after a 3-day incubation was analyzed with the Annexin V assay by flow cytometry. Data are presented as the means $\pm \mathrm{SD}, \mathrm{n}=10$. $^{* *} \mathrm{P}<0.05$ represent ZGDHu-1 with fludarabine vs. ZGDHu-1 alone; ${ }^{\mathrm{NS}}$ No significant difference between the ZGDHu-1 and ZGDHu-1 + GSH; ${ }^{*} \mathrm{P}<0.05$ between treatment and control. Control, $0.05 \%$ DMSO (v/v) drug-free medium; F, fludarabine; Z, ZGDHu-1. (E) Data revealed that GSH partly participates in the ZGDHu-1-induced apoptosis of CLL cells. Representative data of 10 independent experiments are shown following exposure to ZGDHU-1, fludarabine alone or in combination with ZGDHu-1 for 3 days in the absence of or in the presence of $100 \mu \mathrm{M} \mathrm{GSH}$.

ROS scavenger glutathione (GSH) was also investigated as to whether it could suppress the apoptosis of CLL cells mediated by ZGDHu-1 or the combination of ZGDHu-1 and fludarabine. Pretreatment with GSH $(100 \mu \mathrm{M})$ for $2 \mathrm{~h}$ significantly blocked ZGDHu-1-induced ROS generation (Fig.4 A and C, P<0.05). In contrast, GSH did not inhibit the pro-apoptotic effects of ZGDHu-1 on the CLL cells (Fig. 4D and E). Overall, these results suggest that $\mathrm{ZGDHu}-1$ may induce the apoptosis of CLL cells by altering the ROS level.
Effect of the combination of ZGDHu-1 and fludarabine on the mitochondrial pathway through $\Delta \Psi m$. To further investigate whether ZGDHu-1 induces CLL cell apoptosis through the mitochondrial pathway, we analyzed the $\Delta \Psi$ m after ZGDHu-1 and/or fludarabine treatment by FACS analysis. After the CLL cells were treated with ZGDHu-1 and/or fludarabine on day 3 and 5, then the CLL cells stained with JC-1 $(10 \mu \mathrm{mol} / \mathrm{l})$ were detected for the $\Delta \Psi \mathrm{m}$ level. As shown in Fig. $5 \mathrm{~B}$, the percentage of cells with low $\Delta \Psi \mathrm{m}$ in the ZGDHu-1 + fludara- 
A

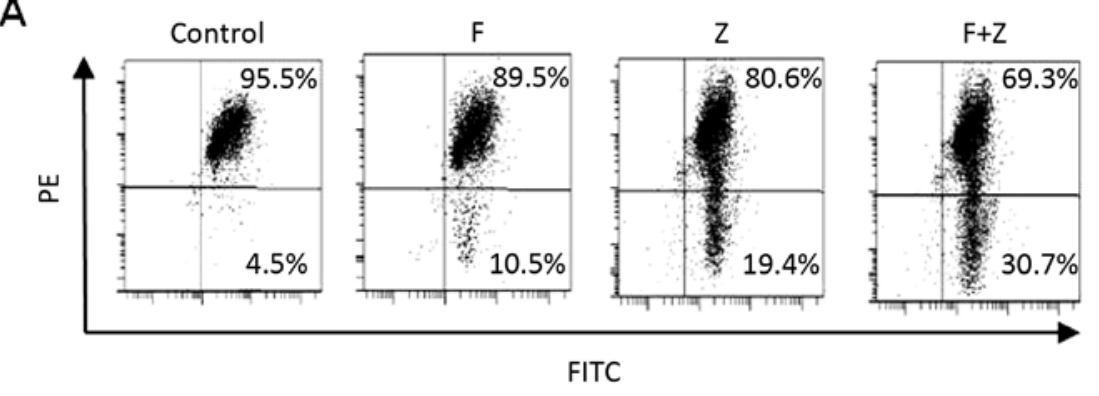

B

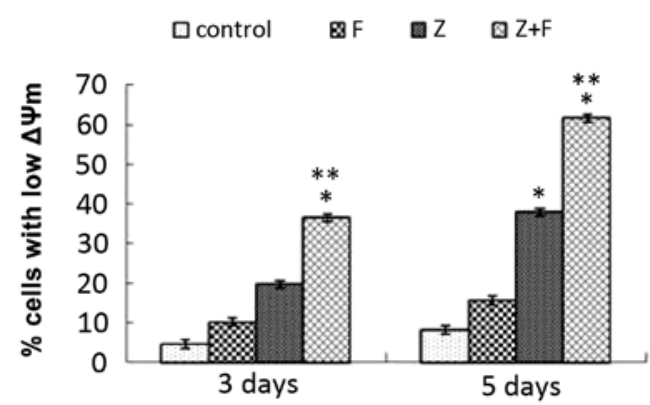

Figure 5. Effect of ZGDHu-1 and fludarabine on the $\Delta \Psi \mathrm{m}$ of CLL cells. (A) Representative data of 10 independent experiments are shown following exposure to ZGDHu-1 alone or the combination of ZGDHu-1 and fludarabine for 3 days. Control, $0.05 \%$ DMSO drug-free medium (v/v); F, $1 \mu \mathrm{g} / \mathrm{ml}$ fludarabine; Z, $100 \mathrm{ng} / \mathrm{ml}$ ZGDHu-1. (B) Purified CLL cells were cultured in $0.05 \%$ (v/v) DMSO drug-free medium (control) or with ZGDHu-1, fludarabine alone or the combination of ZGDHu-1 and fludarabine for 3 or 5 days. $\triangle \Psi \mathrm{m}$ was measured with JC-1 staining and flow cytometry. The data showed that ZGDHu-1 alone or the combination of ZGDHu-1 and fludarabine caused an early $\Delta \Psi \mathrm{m}$ decrease in a dose-dependent manner. Results are expressed as means $\pm \mathrm{SD}, \mathrm{n}=10 ;{ }^{*} \mathrm{P}<0.05$ vs. respective control, ${ }^{* *} \mathrm{P}<0.05$ represent ZGDHu-1 with fludarabine vs. ZGDHu-1 alone.

bine-treated culture was significantly higher than that of the ZGDHu-1 group and the fludarabine group, indicating that ZGDHu-1 synergistically acts with fludarabine inducing CLL cell apoptosis through the mitochondrial pathway.

Effect of the combination of ZGDHu-1 and fludarabine on the mitochondrial pathway through the Bcl-2 family. As known, Bcl-2 family proteins play an important role in the control of membrane permeability of mitochondria and caspase activation (23). The expression levels of anti-apoptotic factor Bcl-2 and pro-apoptotic Bax factor in CLL cells on day 3 and 5 were detected by FACS (Fig. 6A) and western blot analysis (Fig. 6B). Following exposure to ZGDHu-1 alone and in combination with fludarabine, the Bcl-2 expression was significantly decreased while the Bax expression was not changed. However, when CLL cells were exposed to fludarabine alone, the expression of Bcl-2 and Bax was not changed (Fig. 6C and D). Overall, these data suggest that ZGDHu-1 induces the apoptosis of CLL cells through the intrinsic mitochondrial pathway, which was different from fludarabine.

Combination of $\mathrm{ZGDHu}-1$ and fludarabine synergistically increases caspase-3 activity in CLL cells. Caspase activation, which is a key event in apoptosis and the downstream signaling pathway of the mitochondrial pathway, also plays an important role in fludarabine-induced cytotoxicity (24). Among the caspase family members, caspase-3 serves as an effector caspase, causing cleavage of a variety of proteins including polyADP-ribose polymerase (PARP), a well-known caspase substrate. Our previous study indicated that caspase-3 is involved in the apoptotic effect induced by ZGDHu-1 in CLL cells (12). In the present study, the minimal caspase-3 activation was observed with fludarabine $(1 \mu \mathrm{g} / \mathrm{ml})$ treatment (Fig. 7). When CLL cells were incubated with a combination of ZGDHu-1 and fludarabine, caspase-3 activation exhibited a 2 -fold increase on day 3 compared to the CLL cells treated either with fludarabine or ZGDHu-1 alone ( $\mathrm{P}<0.01$, Fig. 7A). Furthermore, cleavage of PARP also exhibited a 2-fold increase on day 3 compared to that in the CLL cells treated either with fludarabine or ZGDHu-1 alone (Fig. 7B). When CLL cells were exposed to ZGDHu-1 or ZGDHu-1 + fludarabine in the presence of the broad spectrum caspase inhibitor Z-VAD-fmk, Z-VAD-fmk significantly blocked ZGDHu-1-induced caspase-3 activation as well as the cleavage of PARP (Fig. 7B). Moreover, Z-VAD-fmk pre-treatment also partially attenuated the ZGDHu-1-induced apoptotic effects on the CLL cells by PS externalization (Fig. $3 \mathrm{~A}$ and B), but did not affect the $\Delta \Psi \mathrm{m}$ or the Bcl-2/Bax ratio (data not shown). Subsequently, to evaluate the combined effect of ZGDHu-1 and fludarabine more precisely, the combination index (CI) was calculated where a $\mathrm{CI}<1$ indicates a synergistic effect. Twenty-two patients in the ZGDHu-1 + fludarabine group of the 25 analyzed patients had a synergistic effect (Table II). In addition, CI $=1$ was noted in one patient and $\mathrm{CI}>1$ in two patients, representing an additive or infra-additive effect, respectively. Overall, our data suggest that there is a synergistic effect between these two drugs.

ZGDHu-1-induced apoptosis rate of CLL cells is independent of ZAP-70 and CD38 expression. Traditionally, high CD38 expression, high ZAP-70 expression, immunoglobulin heavy chain genes $(\mathrm{IgVH})$ gene and cytogenetic abnormalities (especially deletions of $11 \mathrm{q}$ and $17 \mathrm{p}$ ) are all associated with the poor prognosis of CLL patients and can define patients who are in an aggressive status $(4,5,25-27)$. To assess whether 
A

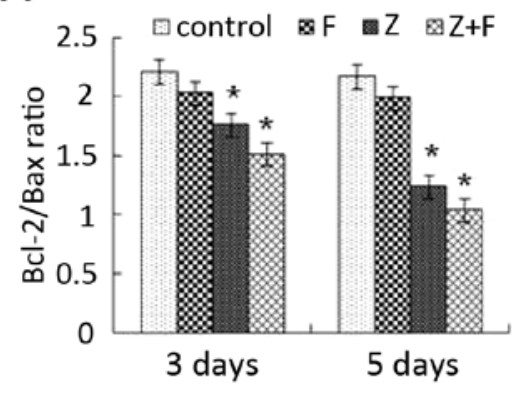

B

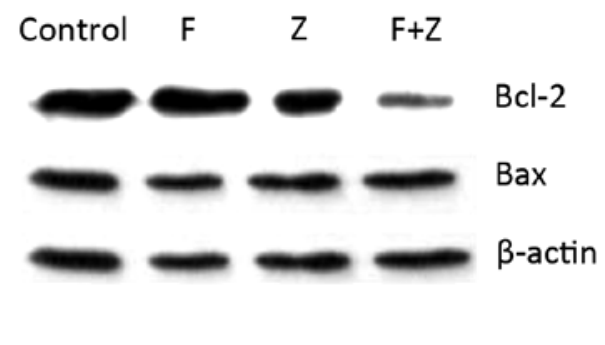

C

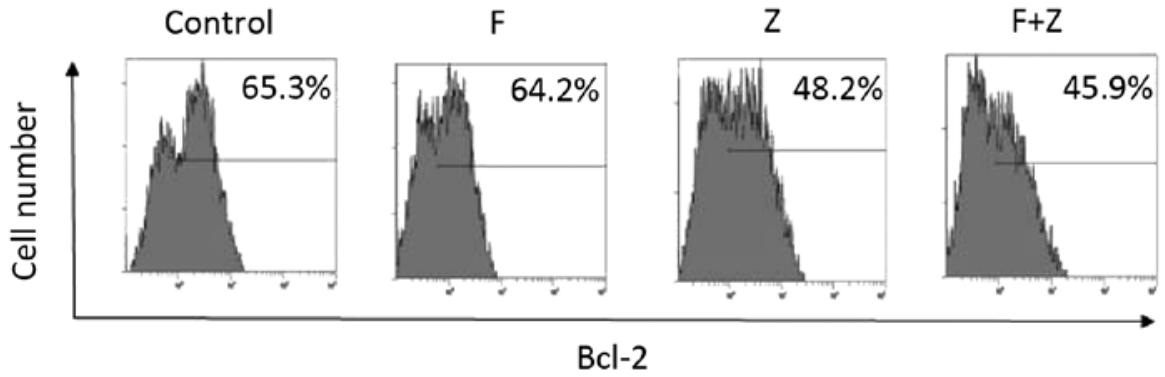

D

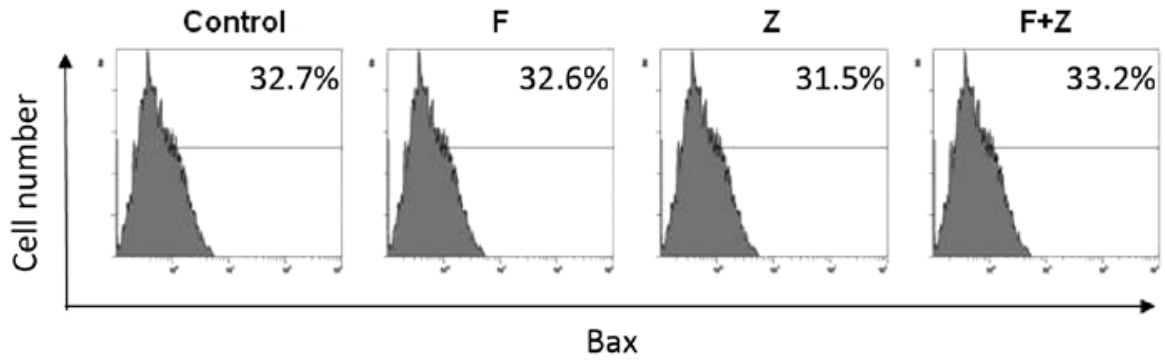

Figure 6. Effect of the ZGDHu-1 and fludarabine on Bcl-2 and Bax levels in CLL cells. Purified CLL cells were cultured with ZGDHu-1, fludarabine alone or the combination of ZGDHu-1 and fludarabine for 3 or 5 days. (A) ZGDHu-1 alone and the combination of ZGDHu-1 and fludarabine induced the negative $\mathrm{Bcl}-2 / \mathrm{Bax}$ ratio modulation. Results are expressed as the means $\pm \mathrm{SD}, \mathrm{n}=10,{ }^{*} \mathrm{P}<0.05$ vs. respective control. (B) The Bax and Bax protein levels after a 3 day treatment in the CLL cells was measured by western blot analysis. Expression of (C) Bcl-2 and (D) Bax in the CLL cells after a 3 day treatment was measured by flow cytometry. Representative FACS histograms of 10 independent experiments are shown. Control, $0.05 \%$ DMSO drug-free medium (v/v); F, $1 \mu \mathrm{g} / \mathrm{ml}$ fludarabine; $\mathrm{Z}, 100 \mathrm{ng} / \mathrm{ml} \mathrm{ZGDHu}-1$.

Table II. Combination indices (CIs) of 25 CLL patients treated with ZGDHu-1 and fludarabine.

\begin{tabular}{lrrrrrrrrrrrrr}
\hline Patient no. & 1 & 2 & 3 & 4 & 5 & 6 & 7 & 8 & 9 & 10 & 11 & 12 & 13 \\
\hline CI & 0.8 & 0.9 & 1.1 & 0.5 & 0.4 & 0.6 & 0.7 & 0.8 & 0.7 & 0.9 & 1.0 & 0.6 & 0.7 \\
\hline
\end{tabular}

\begin{tabular}{lrrrrrrrrrrrr} 
Patient no. & 14 & 15 & 16 & 17 & 18 & 19 & 20 & 21 & 22 & 23 & 24 & 25 \\
\hline $\mathrm{CI}$ & 0.6 & 0.9 & 1.2 & 0.8 & 0.9 & 0.7 & 0.6 & 0.7 & 0.6 & 0.9 & 0.8 & 0.9 \\
\hline
\end{tabular}

The CI calculated for the percentage of active caspase- 3 expression in $\mathrm{CD} 19^{+} \mathrm{CD}^{+}$cells on day 3 in ex vivo cultures with $\mathrm{ZGDHu}-1$, fludarabine and the combination of ZGDHu-1 and fludarabine, separately. CI value $=$ sum of the specific apoptosis of the single drug treatment/specific apoptosis of the combined treatment. The specific apoptosis percentage $=($ drug induced apoptosis - spontaneous apoptosis $) /(100$ - spontaneous apoptosis) $\mathrm{x} 100 \%$. CI $<1, \mathrm{CI}=1$ and $\mathrm{CI}>1$ are defined as synergistic, additive and infra-additive, respectively.

the rate of ZGDHu-1-induced apoptosis is associated with the prognosis of CLL, the apoptotic parameters in ZAP-70 high vs. ZAP-70 ${ }^{\text {low }}$ groups and $\mathrm{CD} 38^{\text {high }}$ vs. CD38 $8^{\text {low }}$ groups were compared. However, a significant difference in the 
B
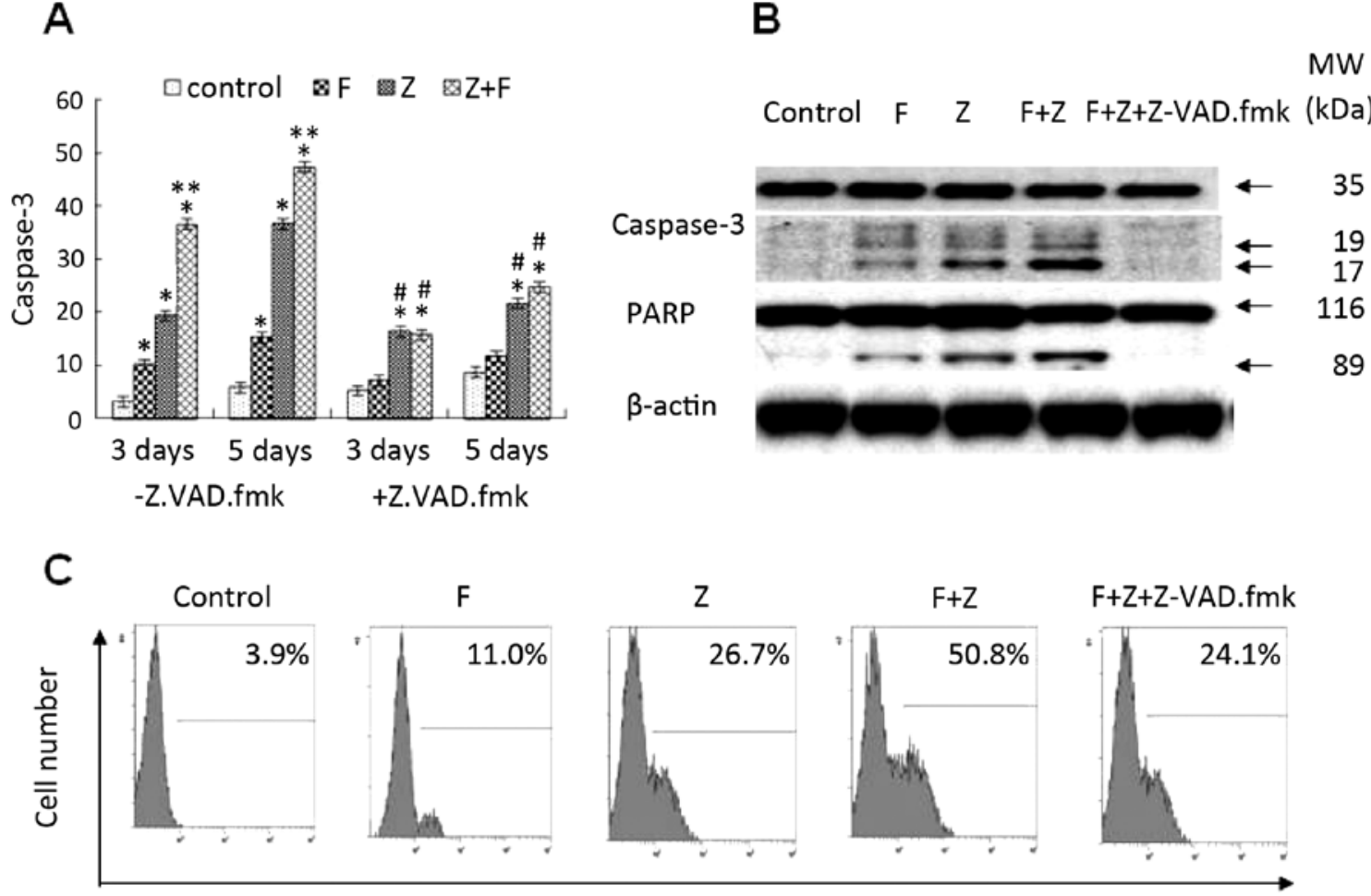

Caspase-3

Figure 7. ZGDHu-1 synergizes with fludarabine to induce caspase-3 activation in CLL cells. (A) Purified CLL cells were cultured in 0.05\% (v/v) DMSO drug-free medium (control) or incubated with ZGDHu-1, fludarabine alone or the combination of ZGDHu-1 and fludarabine in the presence or absence of the broad spectrum caspase inhibitor Z-VAD.fmk for 3 and 5 days. ${ }^{*} \mathrm{P}<0.05$ vs. respective control, ${ }^{* *} \mathrm{P}<0.05$ represent ZGDHu- 1 with fludarabine vs. ZGDHu- 1 alone, ${ }^{\prime P}<0.05$ represent -Z.VAD.fmk vs. +Z.VAD.fmk. (B) Representative western blot analysis for 10 independent experiments demonstrating caspase-3 activation, PARP cleavage, after treatment with ZGDHu-1 and/or fludarabine. (C) Representative active caspase-3 level in CLL cells after a 3 day treatment was measured by flow cytometry. The expression of active caspase-3 was enhanced by these drugs in comparison to control alone. The caspase family inhibitor, Z-VAD-FMK was used as control. Assays were conducted in triplicate ("P<0.01). Control, $0.05 \%$ DMSO (v/v) drug-free medium; F, $1 \mu \mathrm{g} / \mathrm{ml}$ fludarabine; Z, $100 \mathrm{ng} / \mathrm{ml} \mathrm{ZGDHu}-1$.

percentage of caspase-3-positive CLL cells cultured with ZGDHu-1 between the ZAP-70 ${ }^{\text {high }}$ and ZAP-70 ${ }^{\text {low }}$ groups was not found (Fig. 8B), also between the CD38 $8^{\text {high }}$ and CD38 ${ }^{\text {low }}$ patients (Fig. 8C). Moreover, the rate of apoptosis caused by ZGDHu-1 as a single agent or in combination with fludarabine in early (I-II) stage compare to advanced (III-IV) stages disease was assessed (Fig. 8E). In the present study, there was no significant difference between these groups. That is to say, the rate of ZGDHu-1-induced apoptosis was independent of these prognostic markers, such as lymphocytosis, LDH or the 32 -microglobulin level (Fig. 8A and D).

\section{Discussion}

CLL is the most common leukemia in Western countries, characterized by the accumulation of malignant B lymphocytes following the failure to undergo apoptosis. Despite huge progress in treatment, it is still an incurable disease. Currently, purine analogs are widely used for the treatment of CLL and have achieved a higher remission rate $(28,29)$. Fludarabine has been shown to have multiple functions such as interference with DNA synthesis and repair, apoptosis induction and cell cycle regulation in leukemia cells (30). However, the toxic effect of fludarabine such as severe opportunistic infections, myelosuppression and gastrointestinal toxicities including vomiting, nausea and hepatic lesions have been widely reported (6). Thus, reducing the fludarabine toxicity by lowering its dose and exploring new drug are desperately needed. In the present study, we highlight that the use of fludarabine in combination with ZGDHu-1 may reduce the fludarabine dose due to the synergistic effect of the two drugs. The combination of ZGDHu-1 and fludarabine may be useful for the maintenance therapy of CLL patients, as it can sensitize CLL cells to low-dose fludarabine without increasing the risk of long-term side effects on the immune system or other opportunistic infections.

ZGDHu-1, a potential proteasome inhibitor (14), showed significant cytotoxicity on malignant B lymphocytes isolated from CLL patients in a dose-dependent manner, but not on normal B lymphocytes of healthy controls. Moreover, ZGDHu-1 may induce the apoptosis of CLL cells by increasing the mitochondrial membrane permeability, production of ROS, activation of caspase- 3 and a decrease in the Bcl-2/Bax ratio (15). Moreover, the pro-apoptotic effect of ZGDHu-1 was tumor-specific and this effect was not observed on non-leukemic lymphocytes. Furthermore, to the best of our knowledge, this is the first study to demonstrate that ZGDHu-1 may increase the percentage of apoptotic cells in combination with fludarabine and this synergistic effect of ZGDHu-1 with fludarabine was assessed based on the CI. Previous data demonstrated that ZGDHu-1 may induce the apoptosis of CLL cells through the mitochondrial pathway. Moreover, in the present study, the mitochondrial pathway also played an important role in the combination of ZGDHu-1 with fludarabine. As we know, Bcl-2 is regarded as a classical 
A
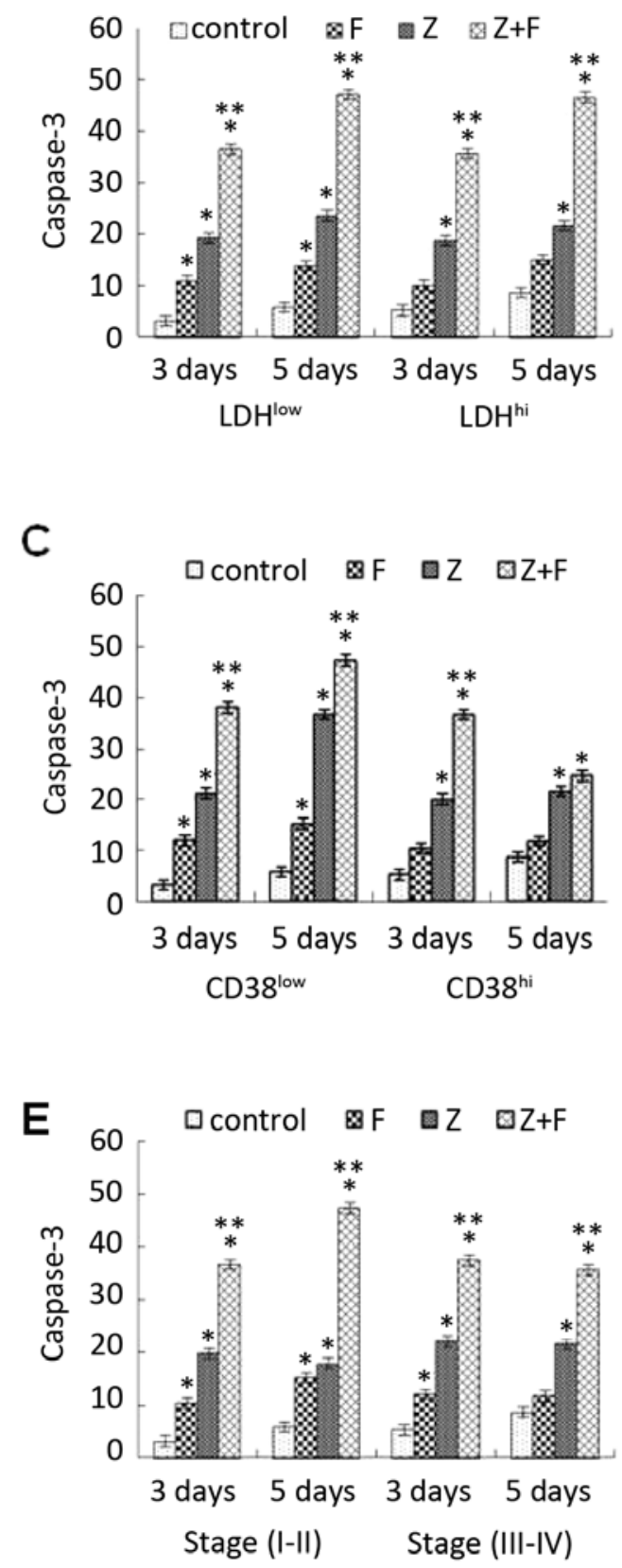

B
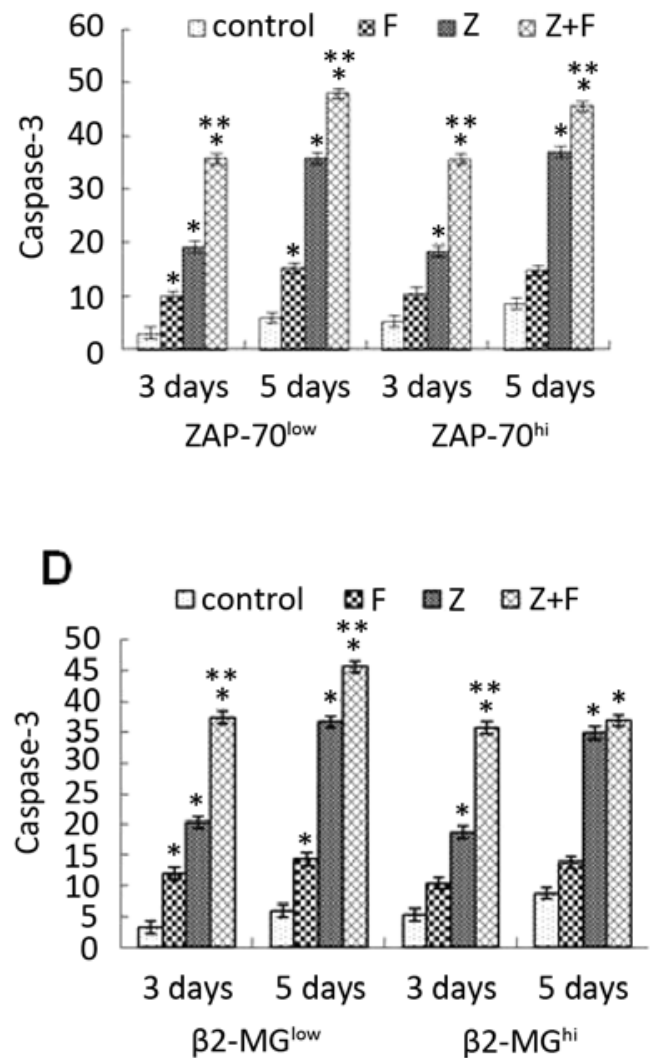

Figure 8. Rate of ZGDHu-1-induced CLL cell apoptosis is independent of ZAP-70 or CD38 expression. The peripheral blood cultures with ZGDHu-1, fludarabine and ZGDHu-1 + fludarabine were analyzed. The concentration of ZGDHu-1 and fludarabine was $100 \mathrm{ng} / \mathrm{ml}$ and $1 \mu \mathrm{g} / \mathrm{ml}$, respectively. Caspase-3 was determined by flow cytometry. (A) ZGDHu- 1 induced a variation in caspase- 3 between $\mathrm{LDH}^{\text {low }}$ and $\mathrm{LDH}^{\text {high }}$ patients. Results are expressed as the means $\pm \mathrm{SD}, \mathrm{n}=10$, ${ }^{*} \mathrm{P}<0.05$ vs. control, respective. (B) ZGDHu-1 induced a variation in caspase-3 between ZAP-70 ${ }^{\text {low }}$ and ZAP-70 ${ }^{\text {high }}$ patients. (C) ZGDHu-1 induced a variation in caspase-3 between $\mathrm{CD} 38^{\text {low }}$ and $\mathrm{CD} 38^{\text {high }}$ patients. (D) The presented data revealed that ZGDHu-1 induced a variation in caspase-3 between $\beta 2-\mathrm{MG}^{\text {low }}$ and $\beta 2-\mathrm{MG}{ }^{\text {high }}$ patients. (E) ZGDHu-1 induced a variation in caspase-3 between stage I-II and stage III-IV patients. All experiments were carried out in triplicate. $\mathrm{n}=7$, $\mathrm{P}<0.05$ vs. respective controls of triplicate wells, ${ }^{* *} \mathrm{P}<0.05$ represent ZGDHu-1 with fludarabine vs. ZGDHu-1 alone. F, indicates $1 \mu \mathrm{g} / \mathrm{ml}$ fludarabine; Z, indicates $100 \mathrm{ng} / \mathrm{ml} \mathrm{ZGDHu}-1$.

marker for the intrinsic apoptosis pathway and the major anti-apoptotic protein of the Bcl-2 family. Overexpression of Bcl-2 may inhibit the apoptosis partly by suppressing ROS generation or by inhibiting the mitochondrial permeability transition (13-15). Overall, compared to fludarabine, ZGDHu-1 had no side effects. Moreover, it had a significant synergistic effect with fludarabine to induce the apoptosis of CLL cells partly through the mitochondrial pathway (31).

As we know, caspase-3 can be cleaved into the 17- and $12-\mathrm{kDa}$ subunits during cleavage and activation (32). In the present study, in CLL cells, ZGDHu-1 induced the complete cleavage of caspase- 3 and this effect was inhibited by the caspase inhibitor Z-VAD-fmk. In addition, caspase-3 was activated partly through the intrinsic pathway. Our results revealed that caspase-3 activation may participate in the synergistic apoptotic combined effect of ZGDHu-1 and fludarabine.

Additionally, several prognostic markers, such as lymphocyte count, LDH elevation, $\beta 2$-microglobulin, IgVH, ZAP-70 and CD38, are widely used for CLL patients $(4,25)$. In the present study, we assessed whether the apoptosis rate caused 
by ZGDHu-1 is different between CLL patients with favorable and unfavorable prognosis. In the present study, the rate of ZGDHu-1-induced apoptosis of CLL cells was independent of ZAP-70 or CD38 expression and the clinical Rai classification status. Furthermore, it did not correlate with lymphocytosis, LDH and $\beta 2$-microglobulin. Overall, our data imply that ZGDHu-1 may be equally effective in CLL patients with both a favorable and poor prognosis.

In conclusion, the present study indicates that ZGDHu-1 may be used as a single agent or in combination with fludarabine for the treatment of CLL patients. One of the mechanisms of ZGDHu-1 synergism with fludarabine appears to be associated with the triggering of caspase- 3 activation that makes CLL cells more susceptible to apoptosis. Thus, ZGDHu-1 combined with fludarabine is a promising treatment strategy for CLL patients.

\section{Acknowledgements}

The present study was supported by the National Natural Science Foundation (no. 30973568), by funding from the key platform funded projects from the Zhejiang Province Health Bureau (no. 2013ZDA005), by the Zhejiang Provincial Program for the Cultivation of High-Level Innovative Health Talents (no. 2012) and by the Zhejiang Province Natural Science Fund (no. LY12H16019).

\section{References}

1. Caligaris-Cappio F and Hamblin TJ: B-cell chronic lymphocytic leukemia: A bird of a different feather. J Clin Oncol 17: 399-408, 1999.

2. Podhorecka M, Halicka D, Klimek P, Kowal M, Chocholska S and Dmoszynska A: Simvastatin and purine analogs have a synergic effect on apoptosis of chronic lymphocytic leukemia cells. Ann Hematol 89: 1115-1124, 2010.

3. Hamblin TJ and Oscier DG: Chronic lymphocytic leukaemia: The nature of the leukaemic cell. Blood Rev 11: 119-128, 1997.

4. Stilgenbauer S: Chronic lymphocytic leukemia: Genetics for predicting outcome. Hematology (EHA Edur Program) 2: 185-190, 2006.

5. Hamblin TJ, Davis Z, Gardiner A, Oscier DG and Stevenson FK: Unmutated $\operatorname{Ig} \mathrm{V}(\mathrm{H})$ genes are associated with a more aggressive form of chronic lymphocytic leukemia. Blood 94: 1848-1854, 1999.

6. Inoue S, Riley J, Gant TW, Dyer MJ and Cohen GM: Apoptosis induced by histone deacetylase inhibitors in leukemic cells is mediated by Bim and Noxa. Leukemia 21: 1773-1782, 2007.

7. Vogler M, Butterworth M, Majid A, Walewska RJ, Sun XM, Dyer MJ and Cohen GM: Concurrent up-regulation of BCL-XL and BCL2A1 induces approximately 1000 -fold resistance to ABT-737 in chronic lymphocytic leukemia. Blood 113: 4403-4413, 2009.

8. Morrison VA: Infectious complications in patients with chronic lymphocytic leukemia: Pathogenesis, spectrum of infection, and approaches to prophylaxis. Clin Lymphoma Myeloma 9: 365-370, 2009.

9. Hu WX, Zhou M, Cai ZB and Yang YZ: Synthesis of new type antineoplastic drug 3, 6-dimethyl-1, 4-dihydro-s-tetrazine-1, 4-dicarboamide. Patent, China, 2004.

10. Rao GW and Hu WX: Synthesis, structure analysis, and antitumor activity of 3,6-disubstituted-1,4-dihydro-1,2,4,5-tetrazine derivatives. Bioorg Med Chem Lett 16: 3702-3705, 2006.

11. Zhou Y, Lv Y, Xu W and Hu W: Determination of proteasome activities with fluorogenic kinetic assays and its application in screening proteasome inhibitor. Chi J Clin Phar Therap 10: $1127-1133,2008$

12. Qiu LN, Zhou YL, Wang ZN, Huang Q and Hu WX: ZGDHu-1 promotes apoptosis of chronic lymphocytic leukemia cells. Int J Oncol 41: 533-540, 2012.
13. Ly JD, Grubb DR and Lawen A: The mitochondrial membrane potential (deltapsi(m)) in apoptosis; an update. Apoptosis 8: 115-128, 2003.

14. Kiss T: Apoptosis and its functional significance in molluscs. Apoptosis 15: 313-321, 2010.

15. Simon HU, Haj-Yehia A and Levi-Schaffer F: Role of reactive oxygen species (ROS) in apoptosis induction. Apoptosis 5: 415-418, 2000

16. Chou TC: Drug combination studies and their synergy quantification using the Chou-Talalay method. Cancer Res 70: 440-446, 2010.

17. Shakir FK, Audilet D, Drake AJ III and Shakir KM: A rapid protein determination by modification of the Lowry procedure. Anal Biochem 216: 232-233, 1994.

18. ten Cate B, Samplonius DF, Bijma T, de Leij LF, Helfrich W and Bremer E: The histone deacetylase inhibitor valproic acid potently augments gemtuzumab ozogamicin-induced apoptosis in acute myeloid leukemic cells. Leukemia 21: 248-252, 2007.

19. Bouzar AB, Boxus M, Defoiche J, Berchem G, Macallan D, Pettengell R, Willis F, Burny A, Lagneaux L, Bron D, et al: Valproate synergizes with purine nucleoside analogues to induce apoptosis of B-chronic lymphocytic leukaemia cells. Br J Haematol 144: 41-52, 2009.

20. Newman A, Clutterbuck RD, Powles RL, Catovsky D and Millar JL: A comparison of the effect of the 3-hydroxy3-methylglutaryl coenzyme A (HMG-CoA) reductase inhibitors simvastatin, lovastatin and pravastatin on leukaemic and normal bone marrow progenitors. Leuk Lymphoma 24: 533-537, 1997.

21. Dimitroulakos J, Nohynek D, Backway KL, Hedley DW, Yeger H, Freedman MH, Minden MD and Penn LZ: Increased sensitivity of acute myeloid leukemias to lovastatin-induced apoptosis: A potential therapeutic approach. Blood 93: 1308-1318, 1999.

22. Rao VA and Plunkett W: Activation of a p53-mediated apoptotic pathway in quiescent lymphocytes after the inhibition of DNA repair by fludarabine. Clin Cancer Res 9: 3204-3212, 2003.

23. Cory S and Adams JM: The Bcl2 family: Regulators of the cellular life-or-death switch. Nat Rev Cancer 2: 647-656, 2002.

24. Sanhes L, Tang R, Delmer A, DeCaprio JA and AjchenbaumCymbalista F: Fludarabine-induced apoptosis of B chronic lymphocytic leukemia cells includes early cleavage of p27kip1 by caspases. Leukemia 17: 1104-1111, 2003.

25. Crespo M, Bosch F, Villamor N, Bellosillo B, Colomer D, Rozman M, Marcé S, López-Guillermo A, Campo E and Montserrat E: ZAP-70 expression as a surrogate for immunoglobulin-variable-region mutations in chronic lymphocytic leukemia. N Engl J Med 348: 1764-1775, 2003.

26. Oscier DG, Thompsett A, Zhu D and Stevenson FK: Differential rates of somatic hypermutation in $\mathrm{V}(\mathrm{H})$ genes among subsets of chronic lymphocytic leukemia defined by chromosomal abnormalities. Blood 89: 4153-4160, 1997

27. Genini D, Budihardjo I, Plunkett W, Wang X, Carrera CJ, Cottam HB, Carson DA and Leoni LM: Nucleotide requirements for the in vitro activation of the apoptosis protein-activating factor-1-mediated caspase pathway. J Biol Chem 275: 29-34, 2000.

28. Van den Neste E, Cardoen S, Offner F and Bontemps F: Old and new insights into the mechanisms of action of two nucleoside analogs active in lymphoid malignancies: Fludarabine and cladribine (Review). Int J Oncol 27: 1113-1124, 2005.

29. Robak T: Therapy of chronic lymphocytic leukemia with purine analogs and monoclonal antibodies. Transfus Apheresis Sci 32: 33-44, 2005.

30. Kobylinska A, Bednarek J, Blonski JZ, Hanausek M, Walaszek Z, Robak T and Kilianska ZM: In vitro sensitivity of B-cell chronic lymphocytic leukemia to cladribine and its combinations with mafosfamide and/or mitoxantrone. Oncol Rep 16: 1389-1395, 2006.

31. Chow KU, Nowak D, Boehrer S, Ruthardt M, Knau A, Hoelzer D, Mitrou PS and Weidmann E: Synergistic effects of chemotherapeutic drugs in lymphoma cells are associated with down-regulation of inhibitor of apoptosis proteins (IAPs), prostate-apoptosis-response-gene 4 (Par-4), death-associated protein (Daxx) and with enforced caspase activation. Biochem Pharmacol 66: 711-724, 2003.

32. Nicholson DW, Ali A, Thornberry NA, Vaillancourt JP, Ding CK, Gallant M, Gareau Y, Griffin PR, Labelle M, Lazebnik YA, et al: Identification and inhibition of the ICE/CED-3 protease necessary for mammalian apoptosis. Nature 376: 37-43, 1995. 